\title{
Conjugate fuzzy QL-implications obtained by the OWA-operator*
}

\author{
Íbero C. K. Benítez Renata H. S. Reiser Adenauer C. Yamin \\ PPGC, CDTEC, UFPel - Pelotas, RS \\ E-mail: \{ickbenitez, reiser, adenauer\}@inf.ufpel.edu.br \\ Benjamín R. C. Bedregal \\ PPSC/PPgEEC - DIMAP - UFRN - Natal, RN \\ E-mail: bedregal@dimap.ufrn.br
}

\begin{abstract}
Fuzzy logic is a powerful theory to make a decision which is usually irresolute for one thing or another, making it difficult to reach a final agreement. So, fuzzy connectives have been extensively studied in computer science and widely used in practical applications such as decision-making pattern recognition also including medical diagnosis, clustering analysis and image processing [3].

Fuzzy implications and aggregation functions are mainly study in this work in order to obtain new representative members in the class of fuzzy QL-operators by analysing related mathematical properties.

Thus, the aggregating fuzzy QL-subimplications are introduced. They are obtained by action of the OWA-operator performed over the family of the product triangular subnorms along with standard fuzzy negation and the probabilistic sum. As the main results, this family of QL-subimplications extend related QL-implications by preserving their corresponding properties. For that, let $U$ be the unitary interval $(U=[0,1])$.

Consider the subconorm $S_{i}: U^{2} \rightarrow U, S_{i}(x, y)=1-\frac{1}{i}(1-x-y+x y)$ for $i \geq 1$, the product t-norm $T: U^{2} \rightarrow U, T(x, y)=x y$ and the standard fuzzy negation $N_{S}: U \rightarrow U, N_{S}(x)=1-x$.

An $n$-tuple of real numbers belonging to $U^{n}$ can be aggregate to a single real number on $U$ by an aggregation function, which is a non-decreasing operator satisfying the following boundary conditions:

$$
A(0,0, \ldots, 0)=0 \text { and } A(1,1, \ldots, 1)=1 .
$$

Let $\sigma: \mathbb{N}^{n} \rightarrow \mathbb{N}^{n}$ be a permutation function ordering the elements: $x_{\sigma(1)} \leq x_{\sigma(2)} \leq \ldots \leq x_{\sigma(n)}$. Let $w_{1}, w_{2}, \ldots, w_{n}$ be non negative weights $\left(w_{i} \geq 0\right)$ such that their sum equals one $\left(\sum_{i=0}^{n} w_{i}=1\right)$. For all $\vec{x} \in U^{n}$, the $n$-ary aggregation function $A: U^{n} \rightarrow U$ called OWA-operator is given as:$$
A(\vec{x})=\sum_{i=0}^{n} w_{i} x_{\sigma(i)}
$$

According with $[4,5]$, a function $I: U^{2} \rightarrow U$ is a fuzzy subimplication if it satisfies the boundary conditions $I(1,1)=I(0,1)=I(0,0)=1$ together with the left antitonicity and right isotonicity. When a subimplication also verifies $I(1,0)=0$, it is called a fuzzy implication [1]. Additionally, QL-(sub)implication is a fuzzy (sub)implication defined, for all $x, y \in U$ by the following expression:

$$
I_{S, N, T}(x, y)=S(N(x), T(x, y)),
$$

when $T(S)$ is a t-(co)norm and $N$ is a strong fuzzy negation.

${ }^{*}$ This work is supported by the Brazilian funding agencies CAPES and FAPERGS (Ed. PqG 06/2010, under the process number 11/1520-1).
\end{abstract}


Proposition 1. Let $\rho: U \rightarrow U$ be an automorphism [2]. The function $J_{i}: U^{2} \rightarrow U$ and its conjugate fucntion $J_{i}^{\rho}: U^{2} \rightarrow U$, given in Eq.(3) and Eq.(4) respectively, are both fuzzy $Q L$-subimplications:

$$
\begin{aligned}
& J_{i}(x, y)=S_{i}\left(N_{S}(x), T_{P}(x, y)\right)=1-\frac{1}{i}\left(x-x^{2} y\right) \\
& J_{i}^{\rho}(x, y)=S_{i}^{\rho}\left(N_{S}^{\rho}(x), T_{P}^{\rho}(x, y)\right)=\rho^{-1}\left(1-\frac{\rho(x)}{i}(1-\rho(x) \rho(y))\right), \forall x, y \in U .
\end{aligned}
$$

Such family of fuzzy QL-subimplications is referred as $\mathcal{J}$.

Proposition 2. The $Q L$-subimplication $J_{i}, J_{i}^{\rho} \in \mathcal{J}$ verify the properties:

I1 : If $S(N(x), x)=1$ then $I(x, 1) \leq 1$, for all $x \in U$;

I2 $a$ : If $x_{1} \geq x_{2}$ then $I\left(x_{1}, 0\right) \leq I\left(x_{2}, 0\right)$, for all $x_{1}, x_{2} \in U$.

I2 $b$ : If $y_{1} \geq y_{2}$ then $I\left(1, y_{1}\right) \leq I\left(1, y_{2}\right)$, for all $y_{1}, y_{2} \in U$.

In [6], an $k$-ary function $\mathcal{F}_{A}: U^{k} \rightarrow U$ is called as $(A, \mathcal{F})$-operator and given by:

$$
\mathcal{F}_{A}\left(x_{1}, \ldots, x_{k}\right)=A\left(F_{1}\left(x_{1}, \ldots, x_{k}\right), \ldots, F_{n}\left(x_{1}, \ldots, x_{k}\right)\right), \forall x_{1}, \ldots, x_{k} \in U .
$$

Proposition 3. Let $\mathcal{T}_{P}=\left\{S_{i}(x, y)=1-\frac{1}{i}(1-x-y+x y): i \geq 1\right\}$ be a family of $t$-subnorms. The function $\mathcal{T}_{O W A}: U^{2} \rightarrow U$ is a t-subconorm given by Eq.(6) in the following:

$$
\mathcal{S}_{O W A}(x, y)=\sum_{i=0}^{n} w_{i} S_{\sigma(i)}(x, y), \forall x, y \in U .
$$

As the main result, we present the subclass of fuzzy QL-subimplication represented by a t-norm $T_{P}$, the standard negation $N_{S}$ together with a t-subconorm $\mathcal{S}_{P}$, which is obtained by aggregating $n$ fuzzy t-subconorms of the family $\mathcal{S}_{P}$.

Theorem 1. For all $x, y \in U$, the function $\mathcal{J}_{O W A}: U^{2} \rightarrow U$ is a $Q L$-subimplication, given by

$$
\mathcal{J}_{O W A}(x, y)=\mathcal{S}_{O W A}\left(N_{S}(x), T_{P}(x, y)\right) .
$$

Proposition 4. The $Q L$-subimplication $\mathcal{J}_{O W}$ A verify $\mathbf{I k}$ for $\mathbf{k} \in\{1,2 a, 2 b\}$.

Concluding, by Prop. 4, the operator $\mathcal{J}_{O W A}$ preserves the main properties of QL-subimplications. Further work considers the interrelations between this class of subimplications and their possible conjugate functions. Another interesting issue is related to dual constructions of QL-subimplications.

Keywords: OWA-operator, fuzzy $Q L$-(sub)implications; fuzzy (sub)implications.

\section{References}

[1] M. Baczyński and J. Balasubramaniam (2008). Fuzzy Implications (Studies in Fuzziness and Soft Computing) Springer.

[2] H. Bustince, P. Burillo, and F. Soria, Automorphism, negations and implication operators, Fuzzy Sets and Systems, 134 (2003) 209-229.

[3] H. Bustince and G. Beliakov and A. Jurio and R. Mesiar and D. Paternain, Image processing using aggregation functions and Soft Computing techniques, In: Proceedings of FUZZ-IEEE 2013, July 7-10, Hyderabad, pp. 1-8, IEEE Publisher.

[4] G. Cornelis, G. Deschrijver, and E. Kerre, Implications in intuitionistic fuzzy and interval-valued fuzzy set theory: construction, classification and application, International Journal of Approximate Reasoning, 35 (2004) 55-95.

[5] L. Kitainik, "Fuzzy Decision Procedures with Binary Relations", Dordrecht: Kluwer Academic Publisher, 1993.

[6] R. Reiser and B. Bedregal and M. Baczyński, Aggregating Fuzzy Implications, Information Science. 253 (2013), 126-146. 